\title{
Role of the Fourth Transmembrane Segment in TRAAK Channel Mechanosensitivity
}

\author{
Mingfeng Zhang 1,2,\#,*, Fuqiang Yao 1,\#, Chengfang Pan 1,\#, Zhiqiang Yan 1,* \\ ${ }^{1}$ State Key Laboratory of Medical Neurobiology, Human Phenome Institute, Ministry of
}

Education Key Laboratory of Contemporary Anthropology, Collaborative Innovation Center of

Genetics and Development, Department of Physiology and Biophysics, School of Life Sciences,

Fudan University, Shanghai 200438, China

${ }^{2}$ Academy for Advanced Interdisciplinary Studies, Peking University, Beijing 100871, China

Author Contributions: M.Z. conceived the project and designed experiments. M.Z., C.P. and F.Y performed mutagenesis and electrophysiology experiments. M.Z. and Z.Y. wrote the manuscript.

All authors contributed to manuscript preparation.

The authors declare no conflict of interest.

\# M.Z., C.P. and F.Y. contributed equally to this work.

*To whom correspondence should be addressed: mingfengzhang@pku.edu.cn or zqyan@fudan.edu.cn. 


\begin{abstract}
Mechanosensitive ion channels such as Piezo, TRAAK, TRPs and OSCA are important transmembrane proteins that are involved in many physiological processes such as touch, hearing and blood pressure regulation. Unlike ligand-gated channels or voltagegated ion channels, which have a canonical ligand-binding domain or voltage-sensing domain, the mechanosensitive domain and related gating mechanism remain elusive.
\end{abstract} TRAAK channels are mechanosensitive channels that convert a physical mechanical force into a flow of potassium ions. The structures of TRAAK channels have been solved, however, the functional roles of the structural domains associated with channel mechanosensitivity remains unclear. Here, we generated a series of chimeric mutations between TRAAK and a non-mechanosensitive silent TWIK-1 K2P channel. We found that the selectivity filter region functions as the major gate of outward rectification and found that lower part of fourth transmembrane domain (M4) is necessary for TRAAK channel mechanosensitivity. We further demonstrated that upper part of M4 can modulate the mechanosensitivity of TRAAK channel. Furthermore, we found that hydrophilic substitutions of W262 and F121 facing each other, and hydrophobic substitutions of Q258 and G124, which are above and below W262 and F121, respectively, greatly increase mechanosensitivity, which suggests that dynamic interactions in the upper part of M4 and PH1 domain are involved in TRAAK channel mechanosensitivity. Interestingly, these gain-of-function mutations are sensitive to cellpoking stimuli, indicating that cell-poking stimuli generate a low membrane mechanical force that opens TRAAK channels. Our results thus showed that fourth 
transmembrane domain of TRAAK is critical for the gating of TRAAK by mechanical force and suggested that multiple dynamic interactions in the upper part of M4 and PH1 domain are involved in this process.

\section{Introduction}

$\mathrm{K} 2 \mathrm{P}$ channels are indispensable for background leak currents that regulate the membrane potential and excitability of many different cell types. The channel activities are regulated by many physiological factors, such as $\mathrm{pH}$, lipids, temperature, and mechanical force [1]. Fifteen K2P homologues exist in humans and can be structurally and functionally divided into six subfamilies, namely, the TWIK, TREK, TASK, THIK, TALK, and TRESK subfamilies. Among these six subfamilies, three members of the TREK subfamily, the TREK-1, TREK-2, and TRAAK channels, are activated by stimuli from a physical mechanical force [2]. The results of experiments involving TREK channels functionally reconstituted in liposomes together with the lysophospholipids that activate TREK channels indicate that TREK channels sense the physical mechanical force in the lipid membrane environment [3, 4]. Recently, the crystal structures of the human TRAAK channel suggest a lipid seal mechanism for the mechanical force sensing of TRAAK channels [5]. The crystal structures of TREK-2 also reveal that lipids in the channel cavity play an important role in channel gating, which is consistent with the lipid seal mechanism [6].

In this study, we attempted to identify the critical elements in TRAAK channel mechanosensitivity by generating a series of chimeric mutations between 
mechanosensitive TRAAK and a non-mechanosensitive silent TWIK-1 channel. Replacing the pore helix and selectivity filter (PH2-filter), the fourth transmembrane helix (M4) of TRAAK or only M4 of TRAAK with the corresponding residues of TWIK-1 revealed that M4 is indispensable for TRAAK channel mechanosensitivity and that the selectivity filter region is the major gate for outward rectification. Moreover, the results by replacing upper or lower part M4 of TRAAK with corresponding residues of TWIK-1 are consistent with the idea that the lower part of M4 is involved in sensing the mechanical force. Additionally, we found that hydrophilic substitution of W262 and F121, which are facing each other, and hydrophobic substitution of Q258 and G124, which are above and below W262 and F121, respectively, largely increase channel mechanosensitivity, which suggests that dynamic interactions in the upper part of M4 and PH1 domain are involved in mechanical gating of TRAAK channel. Interestingly, the channels with these gain-of-function mutations are more sensitive to cell-poking stimuli than wild-type TRAAK channels, indicating that cell-poking stimuli generate a low membrane mechanical force that opens TRAAK channels.

\section{Chimeras reveal that M4 is indispensable for TRAAK channel mechanosensitivity}

\section{and that the selectivity filter region is the major gate for outward rectification.}

The fifteen K2P homologues display different functions despite their structural and sequence conservation [1]. Structural and sequence conservation provides the possibility of generating chimeric mutations between the different K2P channels. To explore the structural basis of channel mechanosensitivity, we first divided the K2P 
channel into eight segments by structural information: the first transmembrane helix (M1), the two-cap domain (Cap), the first pore helix and selectivity filter (PH1-filter), the second transmembrane helix (M2), the third transmembrane helix (M3), the second pore helix and selectivity filter (PH2-filter), the fourth transmembrane helix (M4) and the C-terminal domain (C-ter) (S1 Fig). We generated the chimeric mutations with a previously reported non-mechanosensitive silent TWIK-1 K2P channel $[7,8]$ and measured the background whole cell currents and negative pressure activated microscopic currents to study the effects of the eight segments on channel activity. Most chimeric mutations showed silent channel characteristics similar to TWIK-1 channels, and some mutations were similar to wild-type TRAAK channels (S2 Fig). Interestingly, replacing the PH2-filter and M4 of TRAAK with the corresponding residues of TWIK1 (2-5 mutation) eliminated both the mechanosensitivity and outward rectification properties, resulting in large linear background potassium currents compared to those of wild-type TRAAK channels (Fig 1). Only replacing M4 of TRAAK with the corresponding residues of the TWIK-1 channel (2-6 mutation) eliminated mechanosensitivity but retained the outward rectification properties, with large voltagedependent background potassium currents compared to those of wild-type TRAAK channels (Fig 1). The two non-mechanosensitive chimeras, the 2-5 and 2-6 mutations, suggested that M4 is critical for channel mechanosensitivity. Additionally, comparison of the 2-5 and 2-6 mutations clearly showed that the PH2-filter plays an important role in outward rectification. These results strongly suggest that the selectivity filter region functions as the major gate for outward rectification and that M4 of TRAAK might 
function as the mechanical force sensing region, which supports the C-type gate activation model [9-11].

The lower part of M4 is necessary for in mechanical gating and the upper part modulates the mechanosensitive currents of TRAAK.

To further study the functional role of M4 in channel mechanosensitivity, we divided M4 into the upper part and the lower part (Fig 2 and S2 Fig). Interestingly, replacing the lower part of the M4 region of TRAAK with the corresponding residues of the TWIK-1 channel (2-6-2 mutation) eliminated the mechanosensitivity and resulted in silent properties (Fig 2A, 2D and 2E), indicating that the lower part of M4 is involved in mechanogating of the channel. Replacing the upper part of the M4 region with the corresponding residues of the TWIK-1 channel (2-6-1 mutation) reduced the half activation pressure threshold $\left(\mathrm{P}_{50}\right)$ from $101.0 \pm 5.4 \mathrm{~mm} \mathrm{Hg}$ to $38.8 \pm 3.8 \mathrm{~mm} \mathrm{Hg}$ (Fig $2 \mathrm{E}$ and $2 \mathrm{~F}$ ), indicating an increase in channel mechanosensitivity. The 2-6-1 mutation also resulted in large voltage-dependent background potassium currents compared to those of the wild-type TRAAK channel (Fig 2B and 2D), suggesting that the mutation breaks some energy barrier for mechanotransduction. We also replaced the M4 upper part of the TREK-1 channel with the corresponding sequences of the TWIK-1 channel, and we found that the chimera had properties similar to the 2-6-1 mutation, which showed increased background potassium channel currents (Fig 2C and 2D). These results suggest that the lower part of M4 might be the core force-sensing domain of TRAAK, and the upper part of M4 modulates the channel activity. 
To study the action of the upper part of M4 in regulating channel activity at the single residue level, we mutated residues in the M4 upper part the TRAAK channel into the corresponding residues of the TWIK-1 channel. The nonconserved sequences of the upper part of M4 comprise a small flexible linker (248-256) and an alpha helix (258265) (Fig 3A and S2 Fig). We found that both the flexible linker (248-256) and the alpha helix (258-265) were involved in channel activity (Fig 3B, 3C and 3F).

We next mutated residues in the alpha helix (258-265) of the TRAAK channel into the corresponding residues of the TWIK-1 channel step by step and found that Q258K and I265L both increased background potassium currents and mechanosensitivity (Fig. 3D, $3 \mathrm{E}$ and $3 \mathrm{~F}$ ). These results suggest that multiple elements in the upper part of M4 regulate channel activity.

\section{Hydrophilic substitution of W262 and F121 and hydrophobic substitution of Q258}

\section{and G124 largely increase channel mechanosensitivity.}

Previously, it was reported that W262, which is in the line of Q258 and I265, plays a critical role in TRAAK channel activities [12-14]. The I265L mutation produces a small side chain difference between isoleucine and leucine, while the gain-of-function mutations W262S and Q258K produce relatively different side chain properties. To identify the possible mechanisms of W262 and Q258 in channel activity, we mutated W262 and Q258 in the TRAAK channel into the 19 other amino acids. Mutations at W262 led to channels with varying $\mathrm{P}_{50}$ values that correlated with the hydrophobic value of substitutions $\left(\mathrm{R}^{2}=0.5715, \mathrm{p}<0.0001\right)($ Fig $4 \mathrm{~A})$. Substitutions of hydrophilic 
amino acids (W262D, W262E, W262N and W262Q) other than the positively charged amino acids (W262R and $\mathrm{W} 262 \mathrm{~K}$ ) reduced the $\mathrm{P}_{50}$ value, while substitutions of hydrophobic amino acids (W262L, W262V and W262I) led to an increase in $\mathrm{P}_{50}$ (Fig 4A). Mutations at Q258 showed the opposite property as those at W262. The $\mathrm{P}_{50}$ value of Q258 mutations had a negative correlation with the hydrophobic value of substitutions of Q258 $(\mathrm{R} 2=0.7035, \mathrm{p}<0.0001)($ Fig 4B). Substitutions of hydrophobic amino acids (Q258W, Q258L, Q258V, Q258F and Q258I) and the positively charged amino acids (Q258R and Q258K) mainly reduced the $\mathrm{P}_{50}$ value (Fig 4B).

Previous studies suggested that W262 at M4 and G124 at PH1 may interact with each other $[12,13]$. Q258 and W262 are in the upper part of M4, while Q258 is far away from G124 (Fig 4C). If there are multiple dynamic interactions in the upper part of M4 and PH1 domain, other critical sites may exist in this region. To determine the new critical in the PH1 domain, we performed an alanine scan of the PH1 domain. Interestingly, one alanine substitution mutation (F121A) led to a large increase in channel mechanosensitivity (Fig 4D and 4E). We also mutated F121 and G124 in the TRAAK channel to the 19 other amino acids. Interestingly, for F121, the relationship between its hydrophobicity value and $\mathrm{P}_{50}$ was similar to that of $\mathrm{W} 262\left(\mathrm{R}^{2}=0.1476, \mathrm{p}\right.$ $=0.0041)($ Fig $4 F)$, while G124 had a relationship similar to that of $\mathrm{Q} 258\left(\mathrm{R}^{2}=0.2904\right.$, $\mathrm{p}<0.0001$ ) (Fig 4G). The hydrophilic amino acid substitutions of F121 (F121R and F121Q) largely reduced $\mathrm{P}_{50}$ (Fig 4F), while the hydrophobic amino acid substitutions of G124 (G124L, G124F and G124I) reduced the $\mathrm{P}_{50}$ (Fig 4G). These results suggest that hydrophilic substitution of W262 and F121 and hydrophobic substitution of Q258 
and G124 largely increase channel mechanosensitivity, indicating that multiple dynamic interactions in the upper part of M4 and $\mathrm{PH} 1$ domain are important in the gating of TRAAK channels.

\section{The GOF mutations in the upper part of M4 are sensitive to cell-poking stimuli.}

The cell-poking assay imitates touch behavior by using a glass tip to touch the cell, which converts mechanical force into the flow of ions. This assay is popular and successful in studying NompC and Piezo channels [15]. It is difficult to activate the TRAAK channel with a short displacement distance, while using cell-poking stimuli with a long displacement distance will disrupt the whole cell configuration [4]. In the Piezo channel, the $\mathrm{P}_{50}$ threshold is approximately $-30 \mathrm{~mm} \mathrm{Hg}$, and the half activation displacement distance $\left(\mathrm{D}_{50}\right)$ is approximately $5 \mu \mathrm{m}[15,16]$. In our system, the results are consistent with the previously reported results of Piezo channels (Fig 5A). In the wild-type TRAAK channel, the $\mathrm{P}_{50}$ threshold is approximately $100 \mathrm{~mm} \mathrm{Hg}$ (Fig 1F) approximately 3-4 times that of the $m$ Piezo channel, and the $\mathrm{D}_{50}$ is difficult to determine despite the detection of visible cell-poking activated currents at long displacement distances (Fig 5B). In the gain-of-function mutations, the $\mathrm{P}_{50}$ value is approximately $30 \mathrm{~mm} \mathrm{Hg}$ (Fig 4A, 4B, 4F and 4G), which is similar to the $\mathrm{P}_{50}$ threshold of the Piezo channel. We attempted to record the whole cell-poking activated currents of the representative gain-of-function mutations of 2-6-1, F121Q, Q258F and W236E (Fig 5). Comparison to the wild-type TRAAK channels revealed that the gain-of-function mutations make the channels sensitive to cell-poking stimuli (Fig 5), indicating that 
cell-poking stimuli and negative pressure shares a common mechanism to activate mechanosensitive channels, and cell poking has similar effect of actuating mechanosensitive channels with low negative pressure.

\section{Discussion}

Mechanosensitive channels are widely distributed from prokaryotes to eukaryotes and are involved in the responses of many important physiological processes, such as touch hearing and blood pressure regulation [17]. In eukaryotes, only members of the TREK channel subfamilies are mechanosensitive potassium channels negatively regulating the cell excitability [1]. Reported stretch-activated mechanosensitive channels include OSCAs [18], Piezos [15], K2Ps [3, 19, 20], TRPs [21, 22], MscLs [23], and MscSs [24]. OSCA channels are dimer transporter-like family proteins. The M0 and M6 transmembrane helices of OSCA channels may play critical roles in channel mechanosensitivity [18]. Piezo channels are tetramers with long transmembrane helical units (THUs) assembled into a highly curved blade-like structure, and THUs may be involved in Piezo channel mechanosensitivity $[25,26]$. K2P and TRP channels share a similar tetramer topology (one subunit comprises two pore helices and two selectivity filter p-loops in K2P) [5, 27]. The M4 transmembrane helix of K2P channels may serve as the force-sensing domain [5] (Fig 2). MscL channels are pentamers [28]. An iris-like expansion of MscL may be the channel gating mechanism [29]. MscS channels are heptamers [30]. Substantial rotational rearrangement of the three transmembrane helices occurs during channel activation [31]. These proteins, varying from dimer to 
heptamer, have no structural similarities and no recognizable force-sensing domain comparable to the voltage sensor or ligand-binding sites.

However, the electrophysiological properties of OSCA channels show some similarities to TREK channels [18]. Both have outward rectification properties, showing a "valvelike" channel behavior. Additionally, both show large-stretch activated currents and have similar relatively large $\mathrm{P}_{50}$ thresholds compared to that of the well-known mechanosensitive Piezo channel. Mechanosensitive OSCA channels share structural similarity with TMEM16A channels, which are calcium-activated chloride channels [18]. In TMEM16A channels, calcium induces the lower part of the sixth transmembrane helix (M6) to straighten, thus activating the channel [32]. OSCA channels may share a similar mechanism of straightening of the lower M6 region for channel activation [18]. M6 in OSCA and TMEM16A is also reminiscent of M4 in the TRAAK channel, hinting that the upper part of M6 may share functions similar to those of the upper part of M4.

C-type gating is a common means to respond to different physical inputs by changing the ion occupancy of the selectivity filter [9-11]. Large conformational changes of transmembrane helices are not necessary for C-type gating [10]. In K2P channels, slight conformational changes of the cryptic K2P modulator pocket converge at the selectivity filter C-type gate, which strongly affects the channel activity [33], which are consistent our results (Fig. 4C). At this point, when the structure is not available at the superresolution level, functional studies of the channel are most instructive. The outward rectification property of the OSCA and TMEM16A channels and the external ions 
largely affect the channel activity phenotype of TMEM16A [34], similar with TRAAK channel, reminiscent of the "C-type gate" that may exist in OSCA and TMEM16A channels.

\section{Materials and methods}

\section{Molecular biology}

All channel DNA was used in the pIRES2eGFP vector. Human TRAAK and human TREK-1 DNA were obtained from GeneCopoeia, and the sequence was confirmed by Sanger sequencing. All mutations were generated by PCR and a DNA in-fusion protocol. HEK293T cells were maintained in DMEM with 5\% FBS on poly-L-lysinecoated glass cover slips in 12-well plates. Cells were cotransfected using Lipofectamine 3000 (Invitrogen) with a total of $3 \mu \mathrm{g}$ of DNA per 18-mm-diameter cover slip.

\section{Electrophysiology}

HEK293T cells were transfected with plasmids and incubated for 24-36 h before recording. HEK293T cell electrophysiology measurements were performed in the indicated solution. The pipette resistance was 8-10 M $\Omega$. For the cell-attached experiments, the bath solution contained $150 \mathrm{mM} \mathrm{KCl}, 5 \mathrm{mM}$ EGTA and $10 \mathrm{mM}$ Hepes ( $\mathrm{pH}$ 7.2), and the pipette solution contained $142.5 \mathrm{mM} \mathrm{NaCl}, 7.5 \mathrm{mM} \mathrm{KCl}, 5 \mathrm{mM}$ EGTA and $10 \mathrm{mM}$ Hepes $(\mathrm{pH}$ 7.2). For the whole cell experiment, the bath solution contained150 mM NaCl, $5 \mathrm{mM}$ EGTA and $10 \mathrm{mM}$ Hepes ( $\mathrm{pH} 7.2)$, and the pipette solution contained $142.5 \mathrm{mM} \mathrm{KCl}, 7.5 \mathrm{mM} \mathrm{NaCl}, 5 \mathrm{mM}$ EGTA and $10 \mathrm{mM}$ Hepes (pH 
7.2). Recordings of patch-clamped cells were obtained using an Axopatch 700B (Molecular Devices) amplifier (Molecular Devices), filtered at $1 \mathrm{kHz}$ and digitized at 10-100 kHz (Digidata 1440A, Molecular Devices). Negative pressure in the pipette was applied using a Suction Control Pro unit (Nanion) with a stepwise protocol through Clampex software. We used the I/Imax versus negative pressure curve approach to evaluate mechanosensitivity. Imax is the maximum current measured from the excised patch with cell-attached patch clamp settings. Glass probes for the whole-cell poking assay were made from borosilicate glass pipettes that were fire polished until becoming sealed with a tip diameter of approximately $3-4 \mu \mathrm{m}$. The probe was mounted to a piezodriven actuator driven by a controller/amplifier controlled through Clampex software. After the formation of a whole-cell seal, the probe was positioned at $45^{\circ}$ to the cell and gently attached to the cell membrane. All recordings were performed at a room temperature of $22^{\circ} \mathrm{C}$. Data were then analyzed by pClamp10.4 software. All data were acquired from at least five independent cells.

\section{Acknowledgments}

We thank all the Yan lab members for support. The research was supported by funds from the National Key R\&D Program of China Project (Project 2017YFA0103900, 2016YFA0502800) to Z.Y., the Program for Professor of Special Appointment (Eastern Scholar of Shanghai, TP2014008) and the Shanghai Rising-Star Program (14QA1400800) to Z.Y. The National Natural Science Foundation of China (31571083) to Z.Y., Additional support was provided grants from the Young 1000 Talent Program 
of China to Z.Y.

1. Renigunta V, Schlichthorl G, Daut J. Much more than a leak: structure and function of $K(2) \mathrm{p}$ channels. Pflugers Archiv : European journal of physiology. 2015;467(5):867-94. doi: 10.1007/s00424015-1703-7. PubMed PMID: 25791628.

2. Brohawn SG. How ion channels sense mechanical force: insights from mechanosensitive K2P channels TRAAK, TREK1, and TREK2. Annals of the New York Academy of Sciences. 2015;1352:20-32. doi: 10.1111/nyas.12874. PubMed PMID: 26332952.

3. Maingret F, Patel AJ, Lesage F, Lazdunski M, Honore E. Lysophospholipids open the two-pore domain mechano-gated $\mathrm{K}(+)$ channels TREK-1 and TRAAK. The Journal of biological chemistry. 2000;275(14):10128-33. PubMed PMID: 10744694.

4. Brohawn SG, Su Z, MacKinnon R. Mechanosensitivity is mediated directly by the lipid membrane in TRAAK and TREK1 K+ channels. Proceedings of the National Academy of Sciences of the United States of America. 2014;111(9):3614-9. doi: 10.1073/pnas.1320768111. PubMed PMID: 24550493; PubMed Central PMCID: PMC3948252.

5. Brohawn SG, Campbell EB, MacKinnon R. Physical mechanism for gating and mechanosensitivity of the human TRAAK K+ channel. Nature. 2014;516(7529):126-30. doi: 10.1038/nature14013. PubMed PMID: 25471887; PubMed Central PMCID: PMC4682367.

6. Dong $Y Y$, Pike AC, Mackenzie A, McClenaghan $C$, Aryal $P$, Dong $L$, et al. K2P channel gating mechanisms revealed by structures of TREK-2 and a complex with Prozac. Science. 2015;347(6227):1256-9. doi: 10.1126/science.1261512. PubMed PMID: 25766236; PubMed Central PMCID: PMC6034649.

7. Aryal P, Abd-Wahab F, Bucci G, Sansom MS, Tucker SJ. A hydrophobic barrier deep within the inner pore of the TWIK-1 K2P potassium channel. Nature communications. 2014;5:4377. doi: 10.1038/ncomms5377. PubMed PMID: 25001086; PubMed Central PMCID: PMC4102122.

8. Chatelain FC, Bichet D, Douguet D, Feliciangeli S, Bendahhou S, Reichold M, et al. TWIK1, a unique background channel with variable ion selectivity. Proceedings of the National Academy of Sciences of the United States of America. 2012;109(14):5499-504. doi: 10.1073/pnas.1201132109. PubMed PMID: $22431633 ;$ PubMed Central PMCID: PMC3325654.

9. Cordero-Morales JF, Cuello LG, Perozo E. Voltage-dependent gating at the KcsA selectivity filter. Nat Struct Mol Biol. 2006;13(4):319-22. doi: 10.1038/nsmb1070. PubMed PMID: 16532008.

10. Clarke OB, Caputo AT, Hill AP, Vandenberg JI, Smith BJ, Gulbis JM. Domain reorientation and rotation of an intracellular assembly regulate conduction in Kir potassium channels. Cell. 2010;141(6):1018-29. PubMed PMID: 20564790.

11. Cuello LG, Jogini V, Cortes DM, Perozo E. Structural mechanism of C-type inactivation in $\mathrm{K}(+)$ channels. Nature. 2010;466(7303):203-8. doi: 10.1038/nature09153. PubMed PMID: 20613835; PubMed Central PMCID: PMC3033749.

12. Bagriantsev SN, Peyronnet R, Clark KA, Honore E, Minor DL, Jr. Multiple modalities converge on a common gate to control K2P channel function. The EMBO journal. 2011;30(17):3594-606. doi: 10.1038/emboj.2011.230. PubMed PMID: 21765396; PubMed Central PMCID: PMC3181481.

13. Bagriantsev SN, Clark KA, Minor DL, Jr. Metabolic and thermal stimuli control K(2P)2.1 (TREK-1) through modular sensory and gating domains. The EMBO journal. 2012;31(15):3297-308. doi: 10.1038/emboj.2012.171. PubMed PMID: 22728824; PubMed Central PMCID: PMC3411076. 
14. Lolicato M, Riegelhaupt PM, Arrigoni C, Clark KA, Minor DL, Jr. Transmembrane helix straightening and buckling underlies activation of mechanosensitive and thermosensitive K(2P) channels. Neuron. 2014;84(6):1198-212. doi: 10.1016/j.neuron.2014.11.017. PubMed PMID: 25500157; PubMed Central PMCID: PMC4270892.

15. Coste B, Mathur J, Schmidt M, Earley TJ, Ranade S, Petrus MJ, et al. Piezo1 and Piezo2 are essential components of distinct mechanically activated cation channels. Science. 2010;330(6000):55-60. doi: 10.1126/science.1193270. PubMed PMID: 20813920; PubMed Central PMCID: PMC3062430.

16. Albuisson J, Murthy SE, Bandell M, Coste B, Louis-Dit-Picard H, Mathur J, et al. Dehydrated hereditary stomatocytosis linked to gain-of-function mutations in mechanically activated PIEZO1 ion channels. Nature communications. 2013;4:1884. doi: 10.1038/ncomms2899. PubMed PMID: 23695678; PubMed Central PMCID: PMC3674779.

17. Ranade SS, Syeda R, Patapoutian A. Mechanically Activated Ion Channels. Neuron. 2015;87(6):1162-79. doi: 10.1016/j.neuron.2015.08.032. PubMed PMID: 26402601; PubMed Central PMCID: PMC4582600.

18. Zhang $M$, Wang D, Kang Y, Wu J-X, Yao F, Pan C, et al. Structure of the mechanosensitive OSCA channels. Nature Structural \& Molecular Biology. 2018;25(9):850-8. doi: 10.1038/s41594-018-0117-6.

19. Maingret $F$, Fosset $M$, Lesage $F$, Lazdunski $M$, Honore $E$. TRAAK is a mammalian neuronal mechanogated K+ channel. The Journal of biological chemistry. 1999;274(3):1381-7. PubMed PMID: 9880510.

20. Bang H, Kim Y, Kim D. TREK-2, a new member of the mechanosensitive tandem-pore K+ channel family. The Journal of biological chemistry. 2000;275(23):17412-9. doi: 10.1074/jbc.M000445200. PubMed PMID: 10747911.

21. Yan Z, Zhang W, He Y, Gorczyca D, Xiang Y, Cheng LE, et al. Drosophila NOMPC is a mechanotransduction channel subunit for gentle-touch sensation. Nature. 2013;493(7431):221-5. doi: 10.1038/nature11685. PubMed PMID: 23222543; PubMed Central PMCID: PMC3917554.

22. Zhang $M$, Li X, Zheng H, Wen X, Chen S, Ye J, et al. Brv1 Is Required for Drosophila Larvae to Sense Gentle Touch. Cell reports. 2018;23(1):23-31. doi: 10.1016/j.celrep.2018.03.041. PubMed PMID: 29617663.

23. Sukharev SI, Blount $P$, Martinac B, Blattner FR, Kung C. A large-conductance mechanosensitive channel in E. coli encoded by mscL alone. Nature. 1994;368(6468):265-8. doi: 10.1038/368265a0. PubMed PMID: 7511799.

24. Levina N, Totemeyer S, Stokes NR, Louis P, Jones MA, Booth IR. Protection of Escherichia coli cells against extreme turgor by activation of $\mathrm{MscS}$ and $\mathrm{MscL}$ mechanosensitive channels: identification of genes required for MscS activity. The EMBO journal. 1999;18(7):1730-7. doi: 10.1093/emboj/18.7.1730. PubMed PMID: 10202137; PubMed Central PMCID: PMC1171259.

25. Zhao Q, Zhou H, Chi S, Wang Y, Wang J, Geng J, et al. Structure and mechanogating mechanism of the Piezo1 channel. Nature. 2018;554(7693):487-92. doi: 10.1038/nature25743. PubMed PMID: 29469092.

26. Saotome K, Murthy SE, Kefauver JM, Whitwam T, Patapoutian A, Ward AB. Structure of the mechanically activated ion channel Piezo1. Nature. 2018;554(7693):481-6. doi: 10.1038/nature25453. PubMed PMID: 29261642; PubMed Central PMCID: PMC6010196.

27. Jin P, Bulkley D, Guo Y, Zhang W, Guo Z, Huynh W, et al. Electron cryo-microscopy structure of the mechanotransduction channel NOMPC. Nature. 2017;547(7661):118-22. doi: 10.1038/nature22981. PubMed PMID: 28658211; PubMed Central PMCID: PMC5669069.

28. Li J, Guo J, Ou X, Zhang M, Li Y, Liu Z. Mechanical coupling of the multiple structural elements of 
the large-conductance mechanosensitive channel during expansion. Proceedings of the National Academy of Sciences of the United States of America. 2015;112(34):10726-31. doi: 10.1073/pnas.1503202112. PubMed PMID: 26261325; PubMed Central PMCID: PMC4553819.

29. Betanzos M, Chiang CS, Guy HR, Sukharev S. A large iris-like expansion of a mechanosensitive channel protein induced by membrane tension. Nature structural biology. 2002;9(9):704-10. doi: 10.1038/nsb828. PubMed PMID: 12172538.

30. Bass RB, Strop P, Barclay M, Rees DC. Crystal structure of Escherichia coli MscS, a voltagemodulated and mechanosensitive channel. Science. 2002;298(5598):1582-7. doi: 10.1126/science.1077945. PubMed PMID: 12446901.

31. Wang W, Black SS, Edwards MD, Miller S, Morrison EL, Bartlett W, et al. The structure of an open form of an E. coli mechanosensitive channel at 3.45 A resolution. Science. 2008;321(5893):1179-83. doi: 10.1126/science.1159262. PubMed PMID: 18755969; PubMed Central PMCID: PMC3299565.

32. Paulino C, Kalienkova V, Lam AKM, Neldner Y, Dutzler R. Activation mechanism of the calciumactivated chloride channel TMEM16A revealed by cryo-EM. Nature. 2017;552(7685):421-5. doi: 10.1038/nature24652. PubMed PMID: 29236691.

33. Lolicato M, Arrigoni C, Mori T, Sekioka Y, Bryant C, Clark KA, et al. K2P2.1 (TREK-1)-activator complexes reveal a cryptic selectivity filter binding site. Nature. 2017;547(7663):364-8. doi: 10.1038/nature22988. PubMed PMID: 28693035; PubMed Central PMCID: PMC5778891.

34. Peters CJ, Gilchrist JM, Tien J, Bethel NP, Qi L, Chen T, et al. The Sixth Transmembrane Segment Is a Major Gating Component of the TMEM16A Calcium-Activated Chloride Channel. Neuron. 2018;97(5):1063-77 e4. doi: 10.1016/j.neuron.2018.01.048. PubMed PMID: 29478917; PubMed Central PMCID: PMC5860880. 
Fig 1

\section{Chimeras reveal that M4 is indispensable for TRAAK channel mechanosensitivity,} and the selectivity filter region is the major gate for outward rectification.

(A-C) Schematic of a single subunit of the wild-type human TRAAK channel (A), 2-5 (B) and 2-6 (C) mutations (left). Violet segments are the corresponding residues of the TWIK-1 channel. Representative traces of wild-type human TRAAK channel (A) and the channels with the 2-5 (B) and 2-6 mutations (C) with (right) whole cell currents from $-100 \mathrm{mV}$ to $+100 \mathrm{mV}$ with $+20 \mathrm{mV}$ per step in HEK293T cells. (D) Statistical analysis of the current density of wild-type human TRAAK channel (black) and the channels with the 2-5 (red) and 2-6 mutations (blue), as well as the voltage relationships from A-C. Note the outward rectified behavior of the channel with the 2-6 mutation and the ohmic behavior of the channel with the 2-5 mutation. (E) Representative traces of negative pressure-activated wild-type human TRAAK (left) and 2-5 and 2-6 mutation (right) currents at the membrane potential of $+60 \mathrm{mV}$. Negative pressure was applied from $0 \mathrm{~mm} \mathrm{Hg}$ to the negative pressure until the currents reaching the maximum state with steps of $10 \mathrm{~mm} \mathrm{Hg}$. No dominant negative pressure-activated currents were detected with the 2-5 and 2-6 mutations. (F) Negative pressure-activated currents of the wild-type human TRAAK and the 2-5 and 2-6 mutations at the indicated membrane potential were fitted with the Boltzmann equation. $\mathrm{P}_{50}$ is $101.0 \pm 5.4 \mathrm{~mm} \mathrm{Hg}$ for the wild-type human TRAAK channel.

Fig 2 
The lower part of M4 is necessary for in mechanical gating and the upper part of M4 modulate the mechanosensitive currents of TRAAK.

(A-B) Schematic of a single subunit for the 2-6-2 (A) and 2-6-1 (B) mutations (left). Representative traces of 2-6-2 (A) and 2-6-1 (B) mutation (right) whole cell currents from $-100 \mathrm{mV}$ to $+100 \mathrm{mV}$ with $+20 \mathrm{mV}$ steps in HEK293T cells. (C) Representative traces of wild-type human TREK-1 (upper) and TREK-1 (upper part of M4 = TWIK-1) chimera mutation (lower) whole cell currents from $-100 \mathrm{mV}$ to $+100 \mathrm{mV}$ with $+20 \mathrm{mV}$ steps in HEK293T cells. (D) Statistical analysis of the current density of the wild-type human TRAAK (black), 2-6-2 (red), 2-6-1 (blue), wild-type human TREK-1 (violet) and TREK-1 (upper part of M4 = TWIK-1) chimera (yellow) mutation channels and the voltage relationships from A-C. (E) Representative traces of negative pressureactivated 2-6-2 (left) and 2-6-1 mutation (right) currents at the membrane potential of $+60 \mathrm{mV}$. Negative pressure was applied from $0 \mathrm{~mm} \mathrm{Hg}$ until the currents reached the maximum state with steps of $10 \mathrm{~mm} \mathrm{Hg}$. No dominant negative pressure-activated currents were detected for the 2-6-2 mutation. (F) The negative pressure-activated currents of wild-type human TRAAK, 2-6-2 (red) and 2-6-1 (blue) mutation channels at the indicated membrane potential were fitted with the Boltzmann equation. $\mathrm{P}_{50}$ is $101.0 \pm 5.4 \mathrm{~mm} \mathrm{Hg}$ for the wild-type human TRAAK and $38.8 \pm 3.8 \mathrm{~mm} \mathrm{Hg}$ for the 26-1 chimeric mutation.

Fig 3

Multiple elements in the upper part of M4 regulate TRAAK channel activity. 
(A) Schematic in the upper part of M4. The gain-of-function (GOF) chimera mutations are indicated by red boxes. (B-E) Representative traces of negative pressure-activated 248-256, 258-265, Q258K and I265L mutation currents at the membrane potential of $+60 \mathrm{mV}$. Negative pressure was applied from $0 \mathrm{~mm} \mathrm{Hg}$ until the currents reached the maximum state, with steps of $10 \mathrm{~mm} \mathrm{Hg}$. (F) Statistical analysis of the $\mathrm{P}_{50}$ values of indicated mutations at the membrane potential of $+60 \mathrm{mV}$. Statistical significance compared to wild type was calculated using Student's t-test $\left({ }^{*} p<0.05\right)$.

Fig 4

Hydrophilic substitutions of W262 and F121 and hydrophobic substitutions of Q258 and G124 largely increase the channel mechanosensitivity.

(A-B) Statistical analysis of the relationships between $\mathrm{P}_{50}$ and hydrophobicity for the indicated W262 (A) and Q258 (B) substitution mutations. W262R (red) does not show channel activity. The Q258R and Q258K (blue) were removed for the curve fitting. There is a positive correlation between $\mathrm{P}_{50}$ and the hydrophobicity value for W262 ( ${ }^{2}$ $=0.5715, \mathrm{p}<0.0001)$ and a negative correlation for $\mathrm{Q} 258\left(\mathrm{R}^{2}=0.7035, \mathrm{p}<0.0001\right)$. Statistical significance compared to wild type was calculated using Student's t-test (*p $<0.05$ ). (C) Structural alignment of wild-type TRAAK (PDB ID: 3UM7) and G124I mutation (PDB ID: 4RUE) in the upper of M4 and PH1 domain. W262 and F121 show slight conformational changes in the upper of M4 and PH1 domain. (D) Representative traces of negative pressure-activated F121A mutation currents at the membrane potential of $+60 \mathrm{mV}$. Negative pressure was applied from $0 \mathrm{~mm} \mathrm{Hg}$ until the currents reached the maximum state, with steps of $10 \mathrm{~mm} \mathrm{Hg}$. (E) Statistical analysis of the $\mathrm{P}_{50}$ 
values of the indicated mutations at the membrane potential of $+60 \mathrm{mV}$. Statistical significance compared to wild type was calculated using Student's t-test $\left({ }^{*} \mathrm{p}<0.05\right)$. (F-G) Statistical analysis of the relationship between the $\mathrm{P}_{50}$ and hydrophobicity values for the F121 (F) and G124 (G) substitution mutations. F121N, F121P, G124R, G124D, G124E and G124P (red) do not show channel activity. There is a positive correlation between $\mathrm{P}_{50}$ and the hydrophobicity value for F121 $\left(\mathrm{R}^{2}=0.1476, \mathrm{p}=0.0041\right)$ and a negative correlation for $\mathrm{G} 124\left(\mathrm{R}^{2}=0.2904, \mathrm{p}<0.0001\right)$. Statistical significance compared to wild type was calculated using Student's t-test $(* p<0.05)$.

\section{Fig5}

\section{The GOF mutation channels in the upper of M4 and PH1 domain are sensitive to} cell-poking stimuli.

(A-F) Representative traces (upper) and statistical results (lower) of the currents from the cell poking-activated mPIEZO1 channel at the membrane potential of $-60 \mathrm{mV}$ and wild-type human TRAAK, 2-6-1, Q258F, W262E and F121Q mutation channels at the membrane potential of $+60 \mathrm{mV}$. Cells were subjected to a series of mechanical steps via $1 \mu \mathrm{m}$ movements of a stimulation pipette in the whole-cell patch mode. All recordings are representative of at least three separate experiments.

\section{S1 Fig}

The eight segments of human TRAAK and human TWIK-1. The first transmembrane helix (M1), the two-cap domain (Cap), the first pore helix and selectivity filter (PH1filter), the second transmembrane helix (M2), the third transmembrane helix (M3), the 

available under aCC-BY 4.0 International license.

second pore helix and selectivity filter (PH2-filter), the fourth transmembrane helix

(M4) and the C-terminal domain (C-ter) are indicated. F121, G124, Q258 and W262 are highlighted in red.

\section{S2 Fig}

The chimera mutation information and their channel characteristics. 


\section{A}

TRAAK

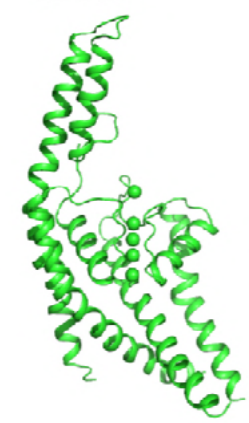

D

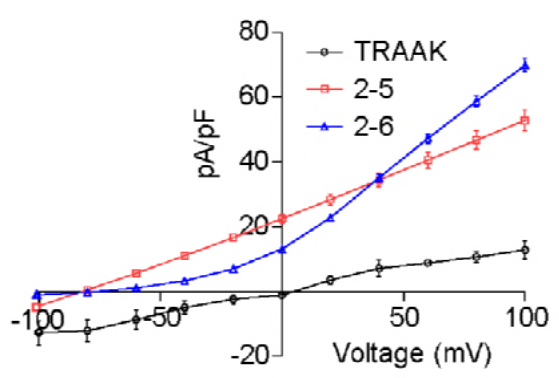

2-5

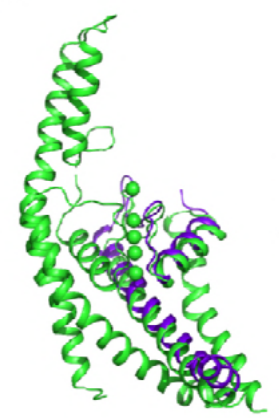

E

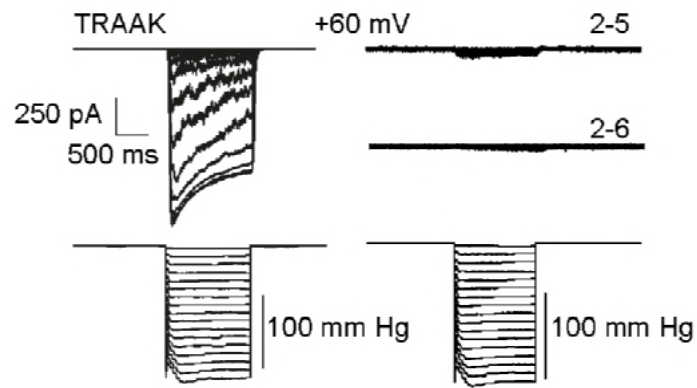

2-6
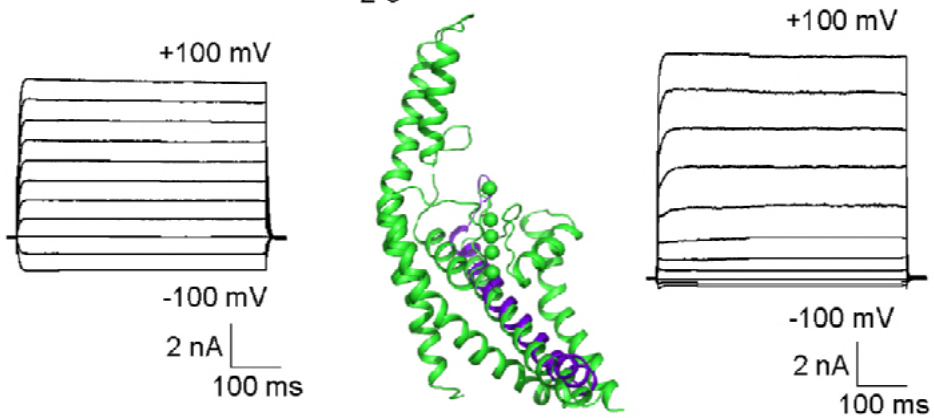

F

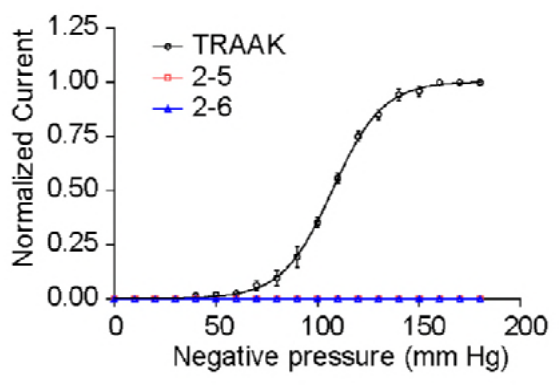




\section{A}

$2-6-2$

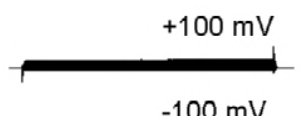

$-100 \mathrm{mV}$

$2 \mathrm{nA}$

$100 \mathrm{~ms}$
2-6-1

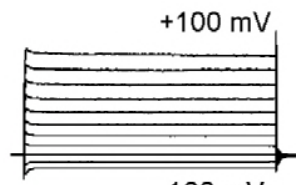

$-100 \mathrm{mV}$

$2 \mathrm{nA}$

$100 \mathrm{~ms}$
TREK-1 $+100 \mathrm{mV}$

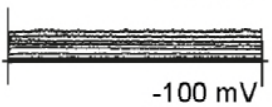

TREK-1-Chimera

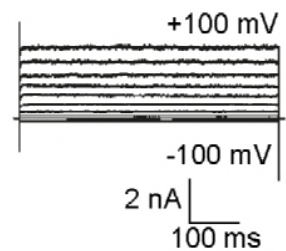

D

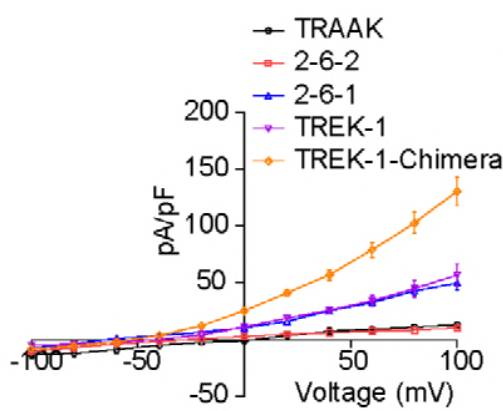

E

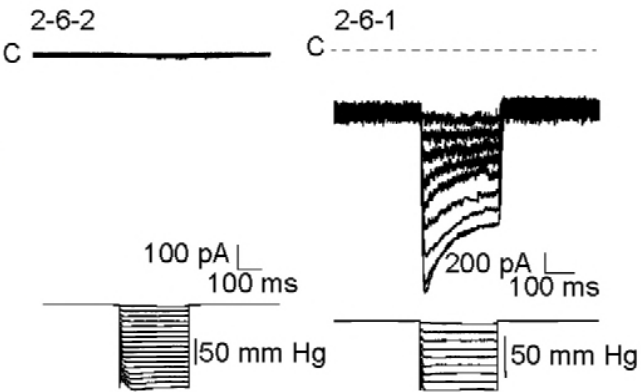

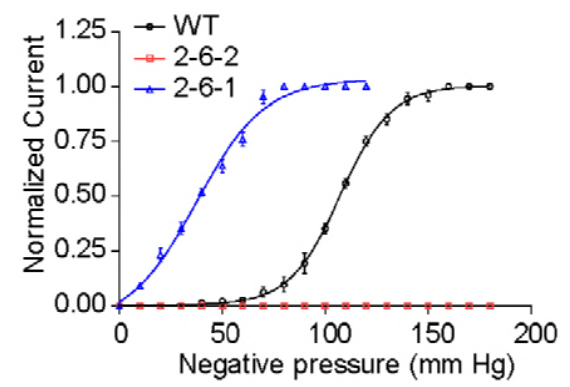


A

B

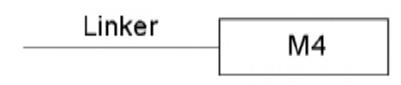

TRAAK: 247 ADPRQDSPA .YQPLWWFWI 266 TWIK-1: 234 EGYNQKFRELYKI GI T CYL 254

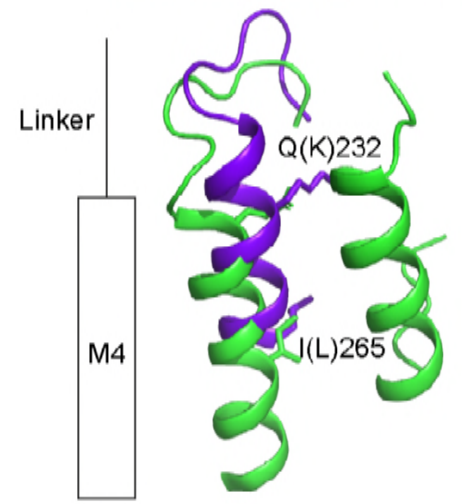

D

Q Q258K

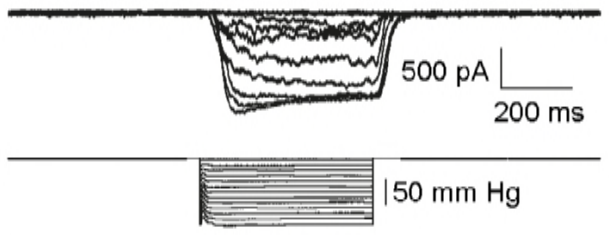

E

$1265 \mathrm{~L}$

C

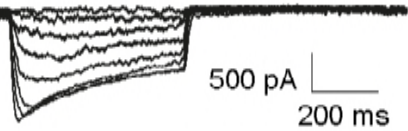

$50 \mathrm{~mm} \mathrm{Hg}$
248-256 (EGYNQKFRELYQPLWWFWI)

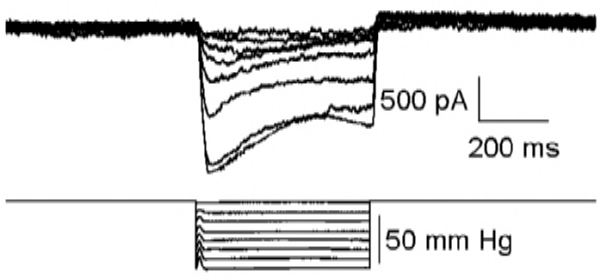

C

258-265 (ADPRQDSPAYKIGITCYL)
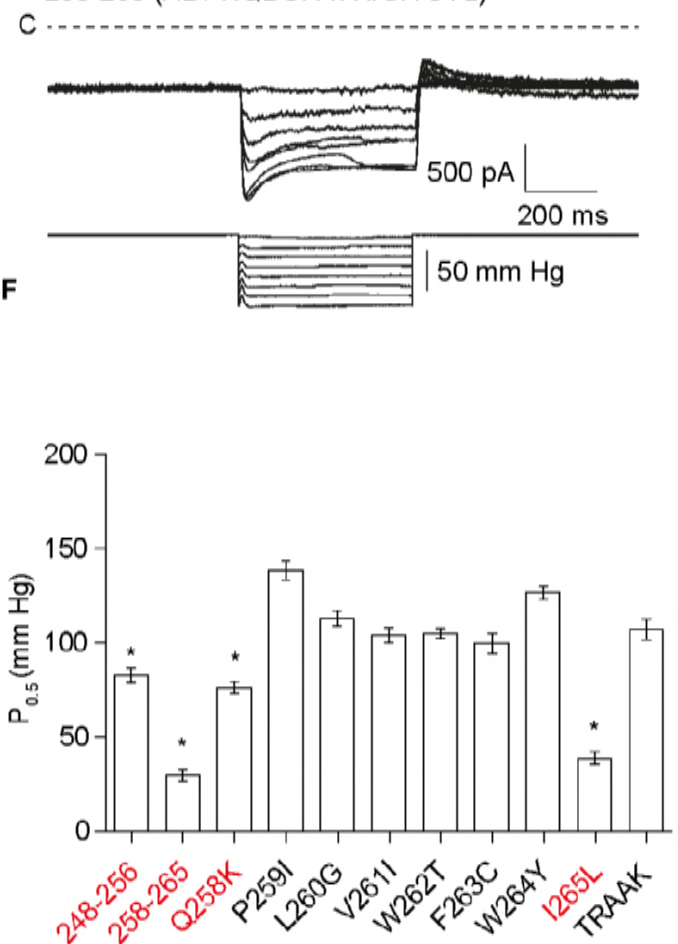


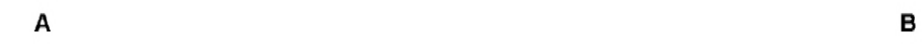

W262

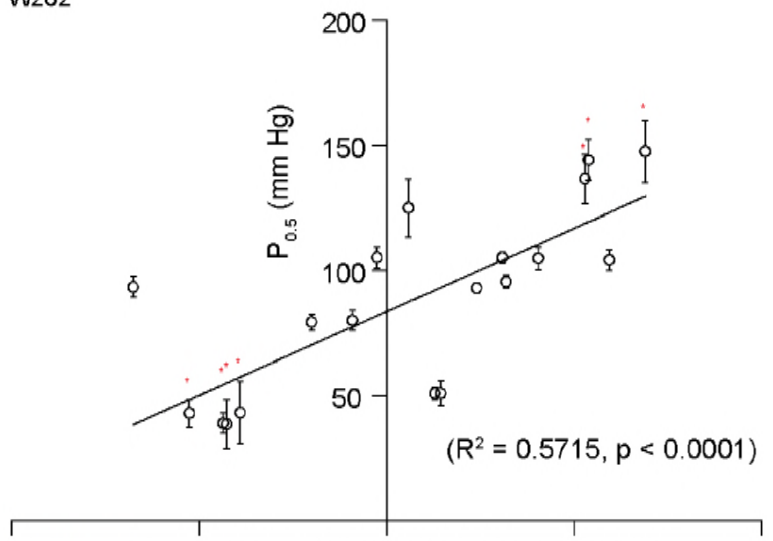

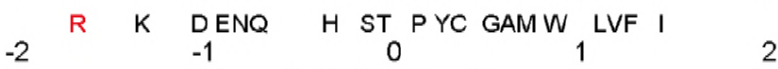

$$
\begin{aligned}
& \text { Hydrophobicity value }
\end{aligned}
$$

C
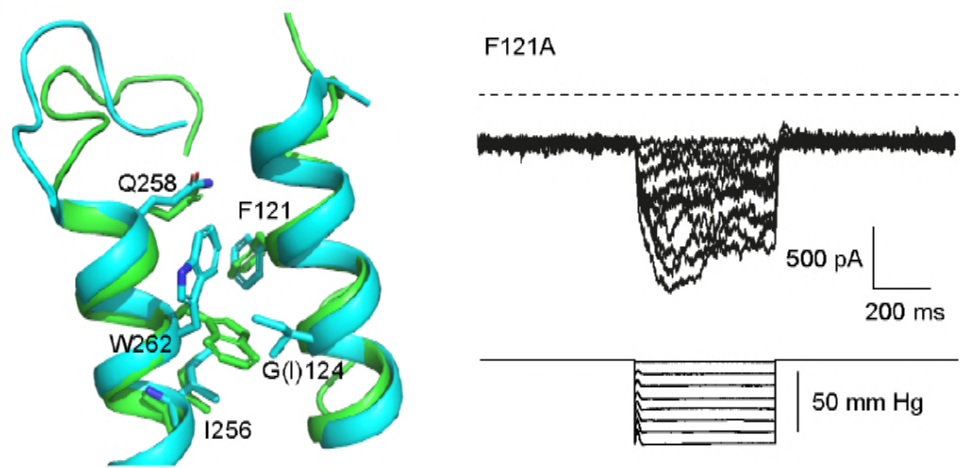

G

F121

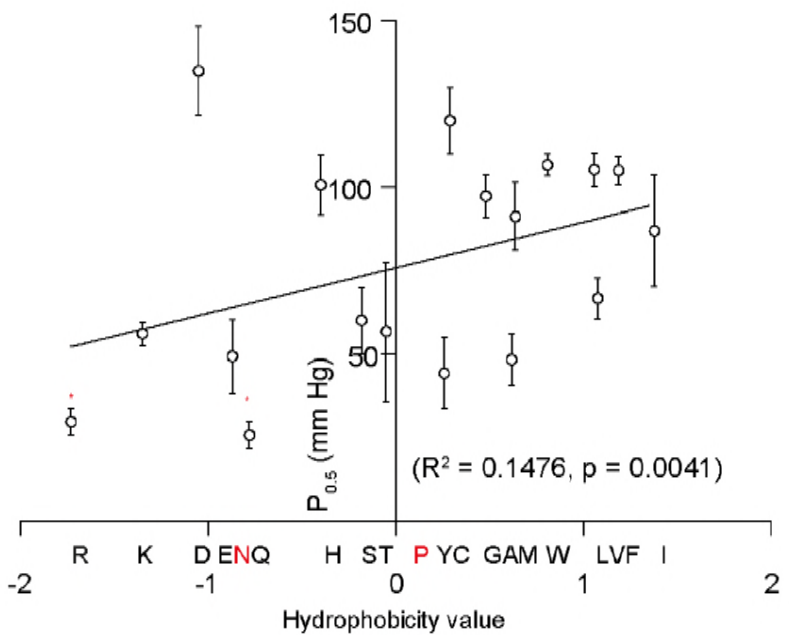

G124
Q258

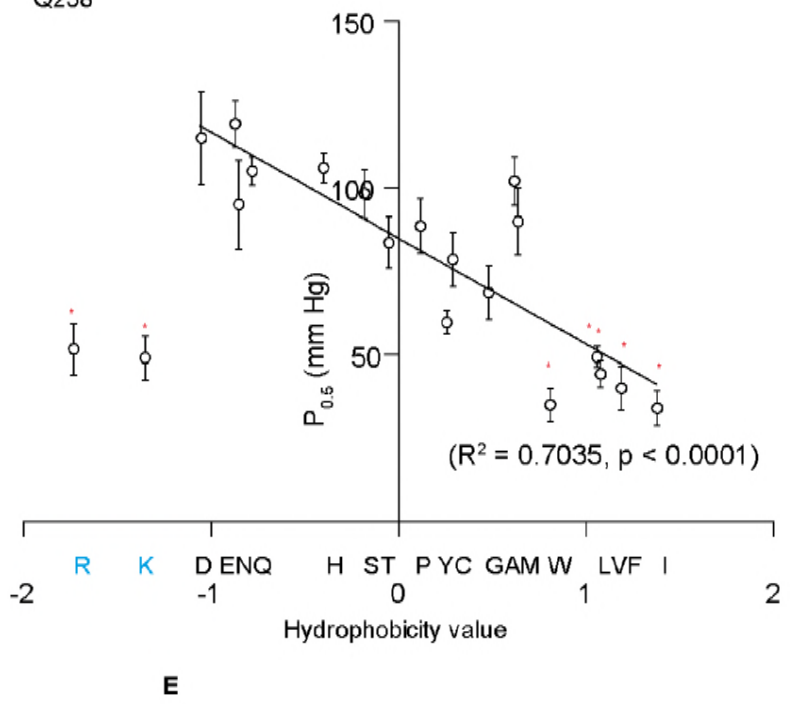

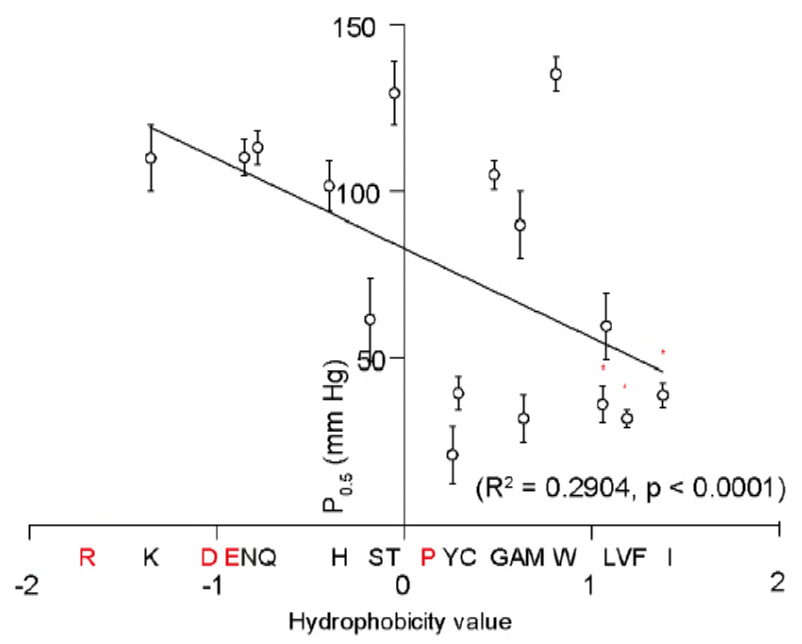

Fig 4 
A
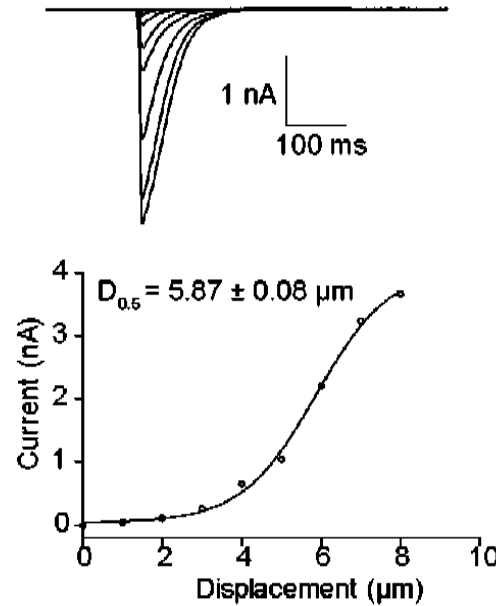

D

Q258F
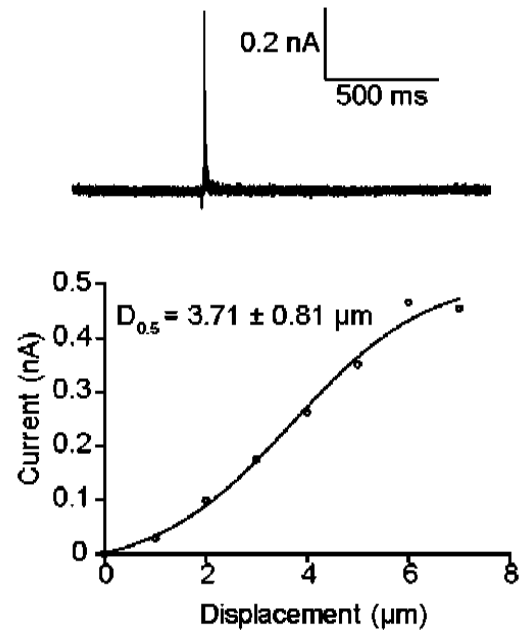

B

$h$ TRAAK
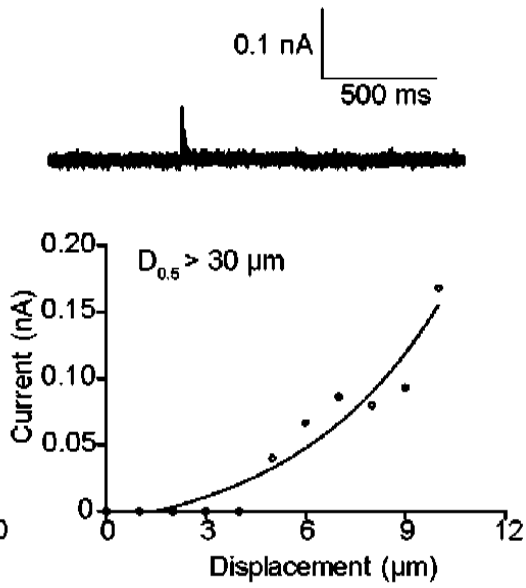

E

W262E
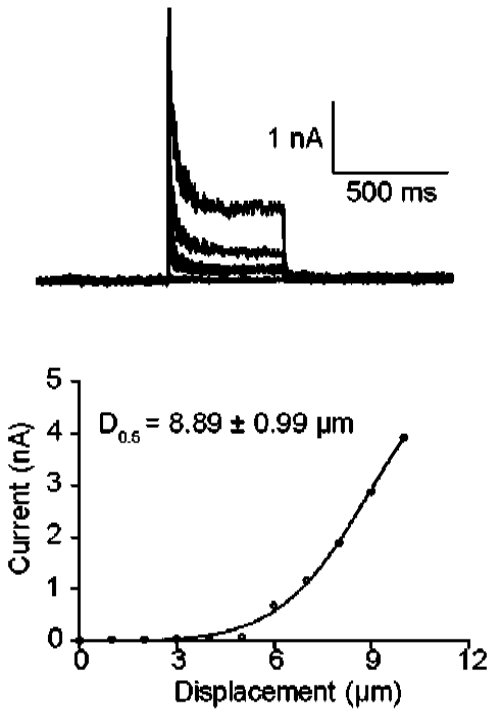

C 2-6-1

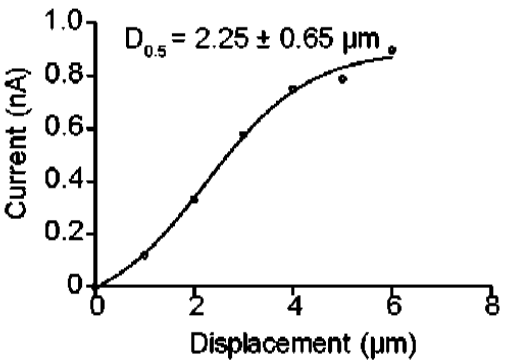

F

F121Q
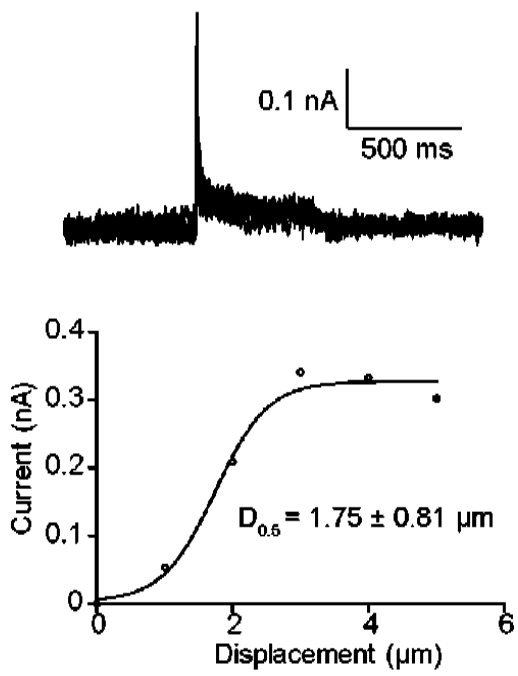
-------------MRSTTLLALIAL-VLLYLVSGALVFRALEQPHEQQAQRELGEVREKF LRAHPCVSDQELGLIIK 63 MLQS LAGS SCVRLVERHRSAWCFGFLVLGYLLYLVFGAVVFS SVELPYEDLLRQELRKLKRRFLEEHECLSEQQLEQFLG 80

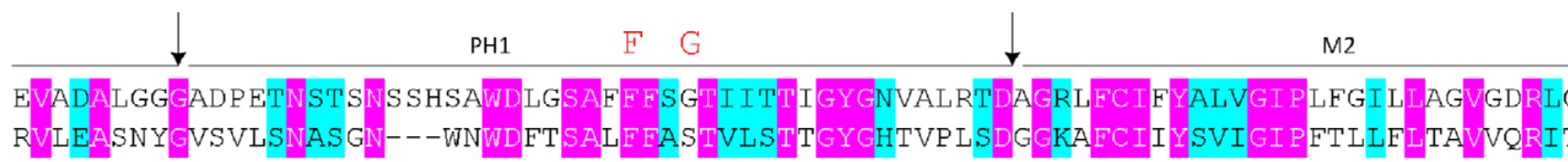

G 143

hTRAAK hTWIK-1

hTRAAK hTWIK-1

hTRAAK hTWIK-1

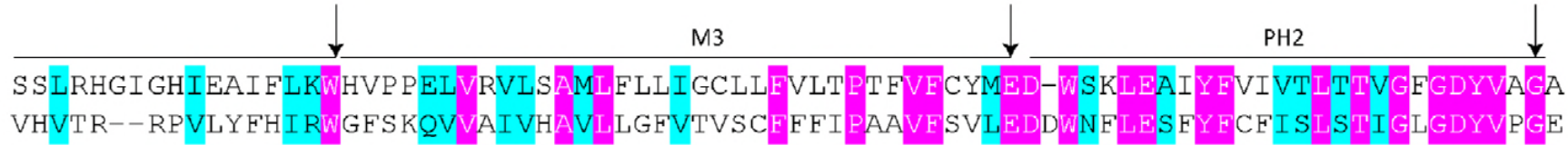

$$
\text { Q } \quad W \quad M 4
$$

DPRQDS-PAYQP LVWFWI LLGLAYFASVLTTI GNWLRVVSRRTRAEMGGLTAQAASWTGTVTARVTQRAGPAAP P PEKEQ GYNQKFRELYKI GITCYLLLGLIAMLVVLETFCELHELKKFRKMFYVK $-\mathrm{KDK}$ Lower part M4

C-ter 


\section{Mutaion Code}

Mutaion region

wild type TRAAK

TRAAK(M1=TWIK-1)

TRAAK (M1, Cap=TWIK-1)

TRAAK(M1, Cap, PH1=TWIK-1)

TRAAK(M1, Cap, PH1, M2=TWIK-1)

TRAAK(M1, Cap, PH1, M2, M3=TWIK-1)

TRAAK(M1, Cap, PH1, M2, M3, PH2=TWIK-1)

TRAAK(M1,Cap, PH1, M2, M3, PH2, M4=TWIK-1)

wild type TWIK-1

2-1

$2-2$

$2-3$

$2-4$

$2-5$

$2-6$

$2-7$

2-6-1

2-6-2
TWIK-1(M1=TRAAK)

TWIK-1(M1, Cap=TRAAK)

TWIK-1(M1, Cap, PH1=TRAAK)

TWIK-1(M1, Cap, PH1, M2=TRAAK)

TWIK-1(M1, Cap, PH1, M2, M3=TRAAK)

TWIK-1(M1, Cap, PH1, M2, M3, PH2=TRAAK)

TWIK-1(M1,Cap, PH1, M2, M3, PH2, M4=TRAAK)

TRAAK(Upper part M4=TWIK-1)

TRAAK(Down part M4=TWIK-1)

wild type TREK-1

TREK-1(Upper part M4=TWIK-1)
Background currents (+100 mV)

Too small

Too small

Too small

No visible currents

No visible currents

No visible currents

No visible currents

No visible currents

No visible currents

No visible currents

No visible currents

No visible currents

No visible currents

$55 \pm 4 \mathrm{pA} / \mathrm{pF}$

$69 \pm 9 \mathrm{pA} / \mathrm{pF}$

Too small

$87 \pm 9 \mathrm{pA} / \mathrm{pF}$

No visible currents

$46 \pm 3 \mathrm{pA} / \mathrm{pF}$

$118 \pm 8 \mathrm{pA} / \mathrm{pF}$
P50 (+60 mV)

$101.0 \pm 5.4 \mathrm{~mm} \mathrm{Hg}$ $110.2 \pm 7.9 \mathrm{~mm} \mathrm{Hg}$ $121.6 \pm 11.2 \mathrm{~mm} \mathrm{Hg}$ No visible currents No visible currents No visible currents No visible currents No visible currents No visible currents No visible currents No visible currents No visible currents No visible currents No visible currents No visible currents $113.9 \pm 8.5 \mathrm{~mm} \mathrm{Hg}$

$38.8 \pm 3.8 \mathrm{~mm} \mathrm{Hg}$ No visible currents

$79.7 \pm 5.8 \mathrm{~mm} \mathrm{Hg}$ $29.7 \pm 3.1 \mathrm{~mm} \mathrm{Hg}$ 